\title{
Joint Propulsion and Cooling Energy Management of Hybrid Electric Vehicles by Optimal Control
}

\author{
Boli Chen, Xuefang Li, Simos A. Evangelou and Roberto Lot
}

\begin{abstract}
This paper develops an optimal control methodology for the energy management (EM) of a series hybrid electric vehicle (HEV) with consideration of ancillary cooling losses, to minimize fuel consumption. Both engine and battery thermal management (TM) models are integrated into the HEV powertrain model as they interact with each other during operation. By collecting all components for propulsion and cooling, a control-oriented model is established, which enables the joint EM and TM optimization problem to be solved simultaneously. Experimental driving cycles are utilized to reveal the impact of the cooling losses on the fuel economy under different driving circumstances. The case study shows the effectiveness of the proposed strategy in finding the optimal power sharing of the hybrid powertrain with consideration of both propulsion and overall cooling requirements. Moreover, a benchmark method based on separately optimized EM and conventional thermostat and PI controlled cooling systems is introduced to verify the solution quality of the proposed approach. It is demonstrated that the proposed method outperforms the benchmark by $0.7 \%$ $\mathbf{2 . 4 9 \%}$ in terms of fuel economy, depending on the driving scenarios.
\end{abstract}

\begin{tabular}{ll} 
CL & \multicolumn{1}{c}{ ABBREVIATIONS } \\
COP & Cooling Load \\
CS & Charge-Sustaining \\
ECMS & Equivalent Consumption Minimization Strategy \\
EM & Energy Management \\
HEV & Hybrid Electric Vehicle \\
ICE & Internal Combustion Engine \\
LHV & Diesel Lower Heating Value \\
NLP & Nonlinear Programming \\
OCP & Optimal Control Problem \\
PL & Propulsion Load \\
PMS & Permanent Magnet Synchronous (Machine) \\
PS & Primary Source of energy \\
SOC & State of Charge \\
SS & Secondary Source of energy \\
TCS & Thermostat Control Strategy \\
TM & Thermal Management \\
VL & Vehicle Load
\end{tabular}

\section{INTRODUCTION}

As a viable solution to address the problems of global warming and natural resources depletion, hybrid electric vehicles (HEVs) have emerged as a leading technology to reduce

B. Chen is with the Dept. of Electronic and Electrical Engineering at University College London, UK (boli.chen@ucl.ac.uk).

X. Li and S. A. Evangelou are with the Dept. of Electrical and Electronic Engineering at Imperial College London, UK (xuefang.li@imperial.ac.uk, s.evangelou@imperial.ac.uk).

R. Lot is with the Dept. of Engineering and the Environment at the University of Southampton, UK (Roberto.Lot@ soton.ac.uk).

This research was supported by the EPSRC Grant EP/N022262/1. emissions and the consumption of fossil fuel. In contrast to conventional vehicles that are only driven by an internal combustion engine (ICE), an HEV usually has an additional electric energy source. Therefore, an appropriate energy management (EM) control strategy is required to determine the power sharing between the two energy sources to minimize fuel consumption. Two main types of EM strategies have been developed in the literature: optimization-based and rule-based control strategies [1]-[6]. The optimization-based methods are essentially based on the formulation of an optimal control problem (OCP), which can be solved by numerous optimization tools, including dynamic programming [1], Pontryagin's Minimum Principle [2], model predictive control [7], [8] and equivalent consumption minimization strategy (ECMS). The main advantage of the methods within this category is that global or local optimality conditions are usually guaranteed. In contrast, rule-based approaches are designed based on empirical control rules that are simple and robust, yet the solutions have no optimality guarantees [9], [10]. Nevertheless, it is worthwhile to note that most of the existing EM strategies, optimization- or rule-based, focus on the propulsion power of the powertrain, while the ancillary energy losses associated with the thermal management (TM) of the energy sources are usually neglected (see, for example, [1]-[14]).

An opportunity therefore exists to design EM control strategies with increased awareness on total power requirements to enhance their fuel efficiency. Beyond this, the ICE and battery thermal management systems are crucial for the safety and reliability of modern HEVs' operation [15]-[21]. Badly controlled energy source temperature may lead to an inefficient operation or even accelerate the aging phenomena. Therefore, the inclusion of ICE and battery thermal models and management systems in the powertrain model is an important step to design practical and more potent overall EM control strategies [22]-[27]. In [23], by considering the influence of the ICE temperature on fuel consumption, a temperature-sensitive optimal control strategy is proposed for a parallel HEV, which is proven to be effective in reducing fuel consumption. However, in that work the energy consumption of the ICE cooling system is still not accounted for. The very recent work [27] proposes an enhanced ECMS by considering the engine cooling losses for parallel HEVs. The results show the superior performance of the cooling-sensitive ECMS over the baseline ECMS. Moreover, the impact of battery cooling losses is investigated in [24], where the battery TM system is integrated into the EM control of a series hybrid electric truck. The result shows that battery cooling energy consumption could increase fuel consumption by as much as $5 \%$. Based on the ECMS strategy, an enhanced EM control approach is 


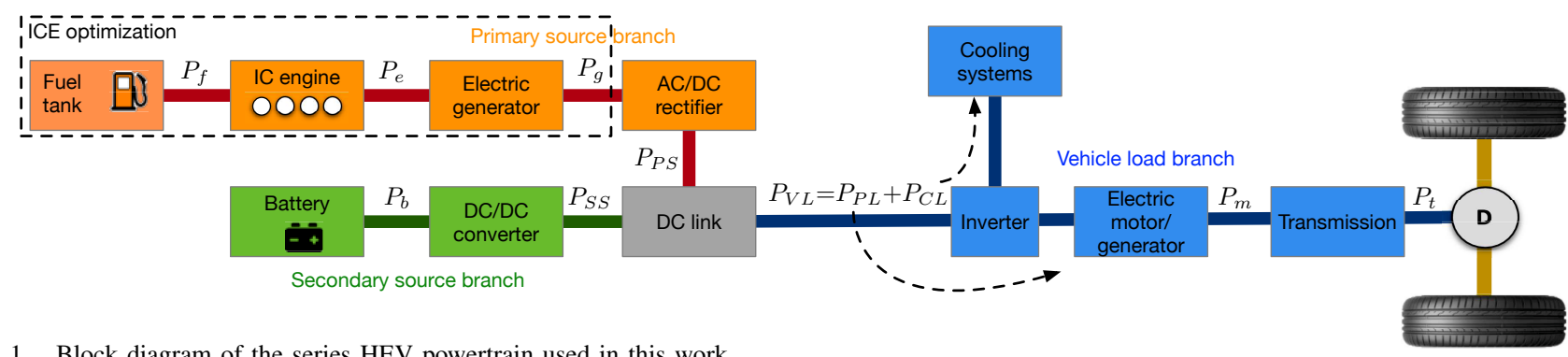

Fig. 1. Block diagram of the series HEV powertrain used in this work.

proposed in [25], which also takes the battery TM system into account. The integrated EM method shows potential fuel savings as compared to the strategy of separately managing the energy and thermal aspects. While this existing body of work, which considers EM with either battery or ICE thermal control, provides some insight into the design of practical EM strategies, it does not consider the mutual influence between the two thermal management systems; the cooling of one source increases the powertrain energy requirements which likely also increases the energy usage of the other source, thereby increasing that source's temperature and hence cooling power requirements, and vice versa. Recently, the work presented in [26] takes both ICE and battery cooling systems into account in a series HEV powertrain, and compares two control mechanisms: 1) the auxiliary cooling components are simultaneously controlled by the EM strategy, 2) the auxiliary systems simply follow an on/off rule independent of the EM algorithm, such that the cooling systems are activated only when the component temperatures are higher than the defined set points. It is shown that the vehicle overall fuel economy can be significantly improved by 1) where the EM and TM are combined. However, the overall EM method is based on a very simple rule-based EM strategy, which lacks optimality.

To the best of the authors' knowledge, the development of an optimization-based EM strategy with consideration of both ICE and battery thermal management systems is still missing. Motivated by this, an optimal control approach is proposed in this work for the EM of a series HEV powertrain with consideration of the losses caused by both ICE and battery cooling systems, which therefore integrates both ICE and battery thermal management systems. Thermal management of the electric machine in the powertrain is not considered because the associated cooling losses are known to be negligible as compared to the losses associated with engine and battery cooling [16]. The main contributions of the present work are summarized as follows: 1) formulation and solving of the EM optimal control problem with joint consideration of propulsion and overall (ICE and battery) cooling requirements in a way that is suitable for numerical optimization, and 2) exposition of the advantages of the joint EM (with TM) optimization as compared to a benchmark EM method that is optimal on the basis of propulsion power but which adopts conventional controllers for the cooling systems. To enable these contributions, a computationally efficient model is employed that describes sufficiently the physical processes involved, which includes: a) models for the ICE and battery cooling systems presented in [16], with suitable (and validated) reduction of their dynamic states, integrated with b) a standard series HEV model from [28], [29], leading to an overall powertrain dynamic model. Moreover, the present study provides some insight into the impact of the auxiliary cooling losses on fuel economy for different experimental driving scenarios.

The rest of the paper is organized as follows. Section II introduces the overall vehicle model including the powertrain, ICE cooling system and battery cooling system. In Section III the optimization problem is formulated with consideration of both the propulsion and cooling requirements, with the details of the benchmark scheme also given. Simulation and comparative results are presented in Section IV. Finally, Section V draws the conclusions of this work.

\section{THe Series HEV Model}

The vehicle studied in this work is a general-purpose passenger car with a series hybrid powertrain, which is sketched in Fig. 1. As it can be seen, this powertrain architecture consists of three branches: primary source (PS) branch, secondary source (SS) branch and vehicle load (VL) branch, which are coupled electrically at the DC-link. The PS branch is formed by an ICE, a permanent magnet synchronous (PMS) generator, and an AC-DC rectifier, which are connected in series. The SS branch includes a battery and a DC-DC converter, and the VL branch includes the propulsion load (PL) subbranch with a DC-AC inverter, a PMS motor/generator, a transmission system with a fixed gear ratio and the wheel load. Thermal management systems of the engine and the battery are also integrated into the VL branch, as the cooling load (CL) subbranch, to maintain the temperature of the energy sources within desired ranges. In the chosen cooling power supply architecture, the cooling power can be provided by either or both of the powertrain sources (PS and SS) as decided by the power split optimization.

Traditional ICE cooling systems consist of a wax based thermostat valve, crankshaft driven water pump and a clutched radiator fan. In particular, the fan can freewheel at low temperatures when cooling is not needed, and the clutch is engaged when the engine temperature increases so that the fan is driven by the engine power to cool the engine. The present work utilizes an ICE cooling system with more advanced components, such as valve, pump and fan, all driven by servo-motors, which have the potential to offer enhanced regulation performance of the coolant fluid flow, and reduced fuel consumption and tailpipe emission as a consequence [30]. On the other hand, the battery cells are cooled by an air conditioning system where the most power consuming actuator 
is the servo-motor driven refrigerant compressor. As such, both engine and battery cooling systems are assumed to be driven by the $\mathrm{AC}$ power from the powertrain inverter, as shown in Fig. 1. The developed battery and engine thermal models are integrated into a conventional model of a series HEV [28], [29], which captures the essential physical characteristics of the powertrain components with a relatively low dynamic order, and which has its main components parameters validated by experimental data. The overall vehicle model consists of 7 states: battery state of charge (SOC), fuel mass and the thermal states, including the engine coolant temperature at engine and radiator outlet, battery cell temperature and the cooling air temperature of engine and battery, respectively. The dynamics of the remaining powertrain components, such as the motor and power converters, are approximated by steady-state representations. Given its low order, this model greatly facilitates the design and implementation of optimization-based EM strategies, which usually demand heavy computational effort.

The driving mission is mostly specified in the literature in terms of driving cycles for the assessment of EM control strategies. When the driving speed profile is known a priori, the driving force $F_{v}$ acting on the wheels is computed as follows:

$$
F_{v}=m_{v} a+F_{T}+F_{D}+m_{v} g \sin \theta,
$$

in which $m_{v}$ is the vehicle mass, $a$ is the vehicle acceleration, $\theta$ is the road slope, and $F_{T}=f_{T} m_{v} g \cos \theta$ and $F_{D}=f_{D} v^{2}$ are the resistance forces respectively due to tires and aerodynamic drag, with $f_{T}$ and $f_{D}$ the rolling resistance and aerodynamic drag coefficients, and $v$ the vehicle speed. The vehicle speed profile (including the acceleration) and road slope are given as inputs to the model according to the tested drive cycles, which will be introduced in Section IV-A.

\section{A. Vehicle Load Branch}

With reference to Fig. 1, the PS power $P_{P S}$ and the SS power $P_{S S}$ are merged at the DC link:

$$
P_{V L}=P_{P S}+P_{S S} \text {. }
$$

The total power $P_{V L}$ received from the energy sources is turned into AC power by the bi-directional inverter, which is modeled as a constant efficiency term $\eta_{i}$. This AC power meets the total power demand from the wheels and the total cooling load. By considering $P_{P L}$ and $P_{C L}$ as the propulsion and cooling loads, respectively, referred at the input of the inverter, it holds that

$$
P_{P L}+P_{C L}=P_{V L}
$$

In particular, $P_{C L}$ is the combined cooling power loss of the ICE $\left(P_{C L E}\right)$ and the battery $\left(P_{C L B}\right)$, both also referred at the input of the inverter:

$$
P_{C L}=P_{C L B}+P_{C L E} .
$$

The PMS motor/generator offers bi-directional energy conversion, according to $P_{m}=\left(\eta_{i} \eta_{m}\right)^{\operatorname{sign}\left(P_{P L}\right)} P_{P L}$. The efficiency of the PMS machine $\eta_{m}$ is described by a static efficiency map of the load torque and the angular speed, as shown in Fig. 2 [28]. The motor speed $\omega_{m}$ is coupled with the speed

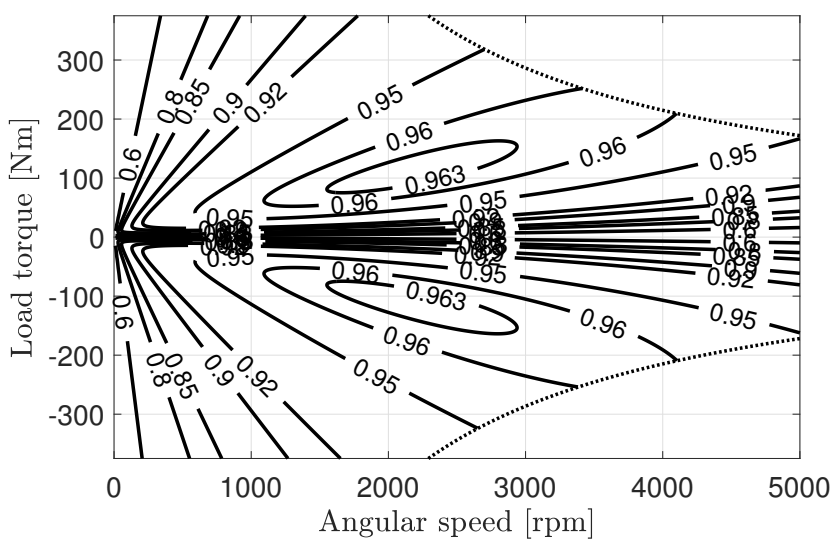

Fig. 2. Efficiency of the reversible PMS machine (generator $=$ positive torque, motor $=$ negative torque) $[28]$. The torque bounds (due to power limitation) are shown by dotted lines. The rated power of the machine is $95 \mathrm{~kW}$.

of the wheels, and consequently the vehicle speed $v$, by a transmission system with a single fixed gear ratio $\gamma$, such that $\omega_{m}=\gamma / r_{w} v$, where $r_{w}$ is the wheel radius. Moreover, the bi-directional power flow through the transmission is modeled by:

$$
P_{t}=\eta_{t}^{\operatorname{sign}\left(P_{m}\right)} P_{m},
$$

with $\eta_{t}$ a constant efficiency factor. By considering the power at the wheels, $P_{v}=F_{v} v$, it is clear that $P_{t}=P_{v}$ when $P_{v} \geq 0$, while when $P_{v} \leq 0$, the regenerated braking power, $P_{t}=P_{v}-P_{h}$, because of the mechanical brakes, $P_{h}$, that are directly applied to the wheels. Due to the series powertrain configuration, the operation of the motor and the transmission is allowed to be isolated from the EM strategy. As such, the power demand (related to propulsion) $P_{P L}$ can be calculated directly from $P_{v}$, as follows:

$$
\left\{\begin{array}{l}
P_{P L}=\frac{P_{v}}{\eta_{i} \eta_{m} \eta_{t}}, \quad \forall P_{v} \geq 0, \\
P_{P L}=\left(P_{v}-P_{h}\right) \eta_{i} \eta_{m} \eta_{t}, \quad \forall P_{v}<0 .
\end{array}\right.
$$

It is also assumed that the energy recovery is not restricted by the braking distribution between front and rear axles, such that all the braking energy (after $P_{h}$ is subtracted) is recoverable.

To sum up, for a given driving cycle, it is possible to compute and use $P_{P L}$ as the input to be followed instead of a speed profile, thus leading to a simplified EM problem framework. The parameters of the VL branch and vehicle force model are listed in Table I.

TABLE I

VL BRANCH AND VEHICLE PARAMETERS

\begin{tabular}{lcc}
\hline \hline Description & Symbol & Value \\
\hline Vehicle mass & $m_{v}$ & $1500 \mathrm{~kg}$ \\
Tyre rolling resistance coefficient & $f_{T}$ & 0.01 \\
Aerodynamics drag coefficient & $f_{D}$ & 0.47 \\
Efficiency of DC-AC inverter & $\eta_{i}$ & 0.96 \\
Efficiency of transmission & $\eta_{t}$ & 0.96 \\
Transmission ratio & $\gamma$ & 3 \\
Wheel radius & $r_{w}$ & $0.3 \mathrm{~m}$ \\
\hline \hline
\end{tabular}

\section{B. Primary Source Branch}

a) Fuel Consumption Model: The ICE in this work represents an Audi 5-Cylinder Turbo Diesel Engine. The 
associated static efficiency map is generated from experimental data available in the Advanced Vehicle Simulator (ADVISOR) [31]. The generator is an identical PMS machine to the motor in the VL branch, but it is only operated with a positive torque (see Fig. 2). The rectifier is simply modeled by a constant efficiency factor $\eta_{r}$. The overall efficiency of this branch is the product of individual component efficiencies, as shown in Fig. 3.

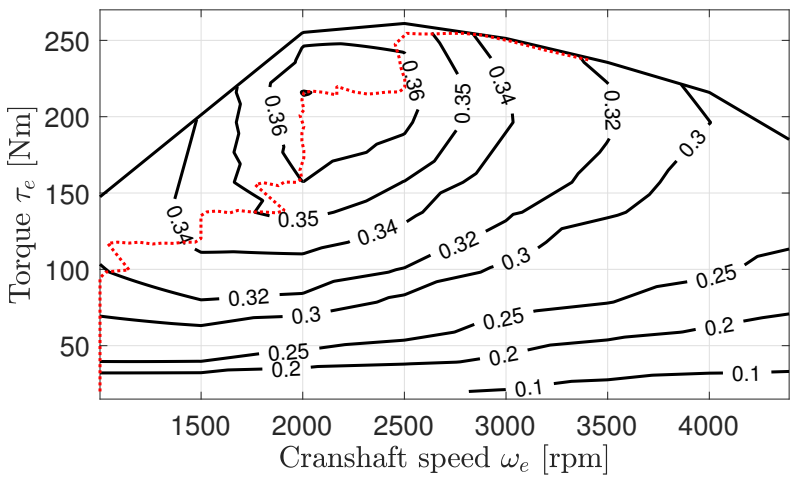

Fig. 3. PS branch combined efficiency map with engine torque and speed operating points. The most efficient torque-speed operating points for different output power values are shown by a dashed curve.

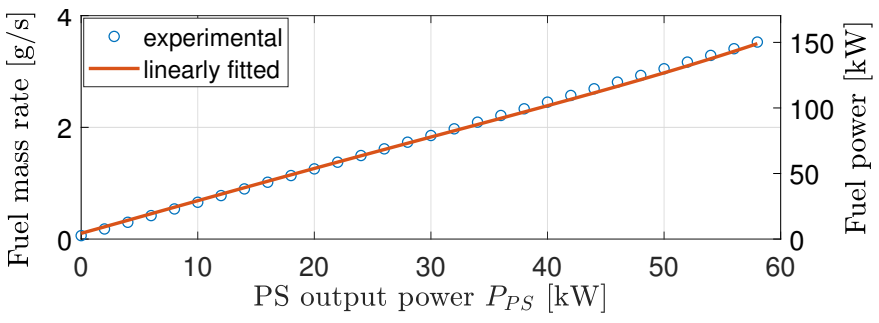

Fig. 4. Fuel mass rate for different PS output power.

The mechanical separation from the wheels allows the PS branch to be constantly operated along the trajectory of the most efficient torque-speed operating points (as shown in Fig. 3). In such a case, the fuel mass rate $q_{f}$ can be fitted approximately as a linear function of the branch output power $P_{P S}$, as shown in Fig. 4. The dynamic equation of the fuel mass $m_{f}$ is therefore given by:

$$
\frac{d}{d t} m_{f}=\dot{m}_{f 0}+\alpha_{f} P_{P S},
$$

in which $\dot{m}_{f 0}$ acts as the idling fuel mass rate, and $\alpha_{f}$ is the coefficient of power transformation. From (7), it is immediate to obtain the fuel power from combustion by:

$$
P_{f}=q_{L H V} \dot{m}_{f 0}+q_{L H V} \alpha_{f} P_{P S}
$$

where $q_{L H V}$ is the diesel lower heating value.

b) Engine Thermal Model: By considering that the efficiencies of the generator and the rectifier are very close to 1 , it can be seen from the PS efficiency map illustrated in Fig. 3 that approximately one third of the chemical power $P_{f}$ can be transformed into engine power output $P_{e}$. The rest of the combustion energy is either taken away by the exhaust waste gas or becomes waste heat that needs to be properly removed by the cooling system. With reference to the data provided in [32], the exhaust gas energy is approximately $30 \%$, while the waste heat energy (from combustion, 32\%, and engine friction, $11.5 \%$ ) is up to $43.5 \%$, of the overall fuel energy. By assuming that the ratio between exhaust gas energy and waste heat energy remains constant at approximately 30:43.5, the waste heat rate that needs to be removed by the engine cooling system can be expressed as:

$$
\begin{aligned}
\dot{Q}_{e} & =\left(P_{f}-P_{e}\right) \sigma \\
& =\left(q_{L H V} \dot{m}_{f 0}+q_{L H V} \alpha_{f} P_{P S}-\frac{P_{P S}}{\eta_{r} \eta_{g}}\right) \sigma,
\end{aligned}
$$

with $\sigma=0.6\left(\approx \frac{1}{\frac{30}{43.5}+1}\right)$ and $\eta_{g}$ the efficiency of the PMS generator (shown in Fig. 2 but varying only with $P_{P S}$ as the PS branch is operated along the trajectory of the most efficient torque-speed operating points shown in Fig. 3). The structure

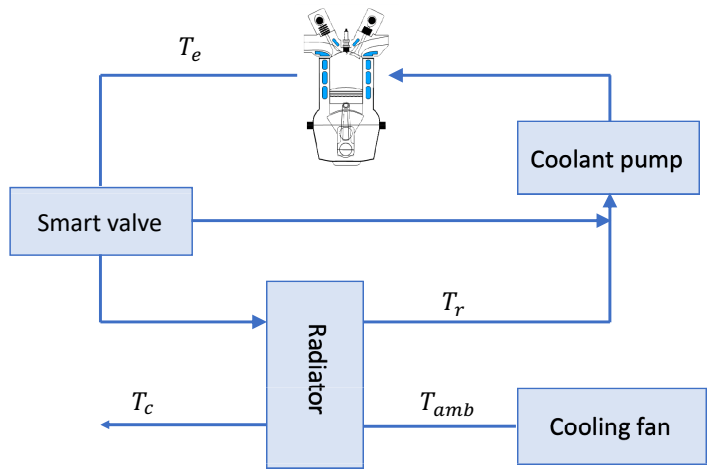

Fig. 5. The schematic diagram of the engine thermal management system.

of the engine cooling system is shown in Fig. 5. As it can be seen, the pump drives the coolant water to circulate within the engine, which then flows into the radiator to be cooled. The heat is discharged to the ambient surroundings by the radiator cooling fan, which controls the air flow through the radiator. The speeds of the fan and the pump are electrically controlled via servo-motors. The smart valve controls the coolant volume that flows through the radiator. The valve is assumed to be fully opened so that all the coolant at engine outlet flows through the radiator.

Similarly to [15], [16], [33], [34], the engine cooling system is modeled based on the convective heat transfer. By starting from the engine thermal model developed in [16], it is further assumed that the coolant temperature at the engine outlet is the same as the engine temperature (i.e. the heat conduction in the engine block is ignored) to reduce the model complexity by one dynamic state; this assumption is validated by simulations. This leads to three differential equations describing the dynamics of the coolant and cooling air temperatures:

$$
\begin{aligned}
& m_{w, e} c_{c} \frac{d T_{e}}{d t}=\dot{Q}_{e}-q_{w} c_{c}\left(T_{e}-T_{r}\right), \\
& m_{w, r} c_{c} \frac{d T_{r}}{d t}=q_{w} c_{c}\left(T_{e}-T_{r}\right)-h_{c a}\left(T_{r}-T_{c}\right), \\
& m_{a, r} c_{a} \frac{d T_{c}}{d t}=h_{c a}\left(T_{r}-T_{c}\right)-q_{a} c_{a}\left(T_{c}-T_{a m b}\right) .
\end{aligned}
$$

This model involves three states, $T_{e}, T_{r}$ and $T_{c}$, the temperatures of the coolant at the engine and at the radiator, and of 
the cooling air, respectively. The ambient temperature $T_{a m b}$ is set to $30^{\circ} \mathrm{C}$ in the present work. The remaining parameters are explained in Table II. $q_{w}$ and $q_{a}$ respectively denote the coolant and cooling air flow rates, which are linearly related to the coolant pump speed, $N_{p}$, and cooling fan speed, $N_{f}$, via the following equations:

$$
q_{w}=\beta_{p} N_{p}, \quad q_{a}=\beta_{f} N_{f},
$$

with the constant factors $\beta_{p}$ and $\beta_{f}$ depending on the specifications of the pump and fan (e.g., pump displacement, fan diameter), and the densities of coolant and cooling air. $N_{p}$ and $N_{f}$ can be manipulated to control the temperature states associated with the cooling system.

According to [16], the power consumed by the pump and fan is proportional to the cube of $q_{w}$ and $q_{a}$, respectively, as described by:

$$
P_{\text {pump }}=K_{p} q_{w}^{3}, \quad P_{\text {fan }}=K_{f} q_{a}^{3},
$$

where $K_{p}$ and $K_{f}$ are two constant coefficients. Finally, the total engine cooling demand $P_{C L E}$ is evaluated by

$$
P_{C L E}=\left(P_{\text {fan }}+P_{\text {pump }}\right) / \eta_{i} .
$$

The engine fuel consumption and thermal model parameter values are presented in Table II [16], [35].

TABLE II

ENGINE BRANCH AND COOLING SYSTEM PARAMETERS

\begin{tabular}{lcc}
\hline \hline Parameter & Symbol & Value \\
\hline Idling fuel mass rate & $\dot{m}_{f 0}$ & $0.11 \mathrm{~g} / \mathrm{s}$ \\
Power transformation coefficient & $\alpha_{f}$ & $0.0583 \mathrm{~g} / \mathrm{kW} / \mathrm{s}$ \\
Diesel lower heating value & $q_{L H V}$ & $42.6 \mathrm{MJ} / \mathrm{kg}$ \\
Efficiency of rectifier & $\eta_{r}$ & 0.96 \\
\hline Coolant specific heat capacity & $c_{c}$ & $4090 \mathrm{~J} / \mathrm{kg} /{ }^{\circ} \mathrm{C}$ \\
Air specific heat capacity & $c_{a}$ & $994 \mathrm{~J} / \mathrm{kg} /{ }^{\circ} \mathrm{C}$ \\
Coolant-air heat transfer coefficient & $h_{c a}$ & $1000 \mathrm{~W} /{ }^{\circ} \mathrm{C}$ \\
Coolant mass at engine & $m_{w, e}$ & $2 \mathrm{~kg}$ \\
Coolant mass at radiator & $m_{w, r}$ & $2 \mathrm{~kg}$ \\
Air mass at radiator & $m_{a, r}$ & $0.3 \mathrm{~kg}$ \\
Cooling air flow rate coefficient & $\beta_{f}$ & $8.7 \times 10^{-4} \mathrm{~kg} / \mathrm{s} / \mathrm{RPM}$ \\
Coolant flow rate coefficient & $\beta_{p}$ & $4.2 \times 10^{-4} \mathrm{~kg} / \mathrm{s} / \mathrm{RPM}$ \\
Cooling fan power coefficient & $K_{f}$ & $78.8 \mathrm{~W} \cdot \mathrm{s}^{3} / \mathrm{kg}$ \\
Coolant pump power coefficient & $K_{p}$ & $86.4 \mathrm{~W} \cdot \mathrm{s}^{3} / \mathrm{kg}^{3}$ \\
\hline
\end{tabular}

\section{Secondary Source Branch}

The battery system is modeled in this section, including both electrical and thermal models. The models are parametrized for the A123 AHR32113 cylindrical cells, which are of $\mathrm{LiFePO}_{4}$ type. The battery consists of 200 identical cells equally divided in 2 parallel strings, with each string formed by 100 cells in series. The cell specifications and the associated battery model parameters for this study are summarized in Table III [16].

a) Battery Electrical Model: The model selection for a Li-ion battery system is subject to a trade-off between accuracy and computational complexity, however for the intended purposes of vehicle powertrain energy management, an equivalent electric circuit model is suitable, for which there is also a rich literature. Therefore, in the present work, the battery is modeled as a series connection of an ideal voltage source (of open-circuit voltage $V_{o c}$ ) and an ohmic resistance (internal
TABLE III

BATTERY ELECTRIC AND THERMAL SYSTEM PARAMETERS

\begin{tabular}{lcc}
\hline \hline Parameters & Symbol & Value \\
\hline Cell capacity & & $4.5 \mathrm{Ah}$ \\
Cell nominal voltage & & $3.3 \mathrm{~V}$ \\
Cell resistance & $n$ & $10 \mathrm{~m} \Omega$ \\
Number of cells & $C_{b}$ & 200 \\
Cell heat capacity & $R_{\lambda}$ & $286.8 \mathrm{~J} /{ }^{\circ} \mathrm{C}$ \\
Cell thermal resistance & $C_{a}$ & $0.65{ }^{\circ} \mathrm{C} / \mathrm{W}$ \\
Cell cooling air heat capacity & $Q_{\max }$ & $0.708 \mathrm{~J} /{ }^{\circ} \mathrm{C}$ \\
\hline Battery capacity & $V_{o c}$ & $9 \mathrm{Ah}$ \\
Open circuit voltage & & $330 \mathrm{~V}$ \\
Discharging/charging C-rate limit & $R_{b}$ & $10 \mathrm{C} / 5 \mathrm{C}$ \\
Internal resistance & $\mathrm{SOC}_{\max }$ & $0.5 \Omega$ \\
SOC upper limit & $\mathrm{SOC}_{\min }$ & 0.8 \\
SOC lower limit & $\eta_{d c}$ & 0.5 \\
Efficiency of DC-DC converter & & 0.96 \\
\hline \hline
\end{tabular}

resistance $R_{b}$ ) [29], in which the battery SOC represents the only state variable, governed by:

$$
\frac{d}{d t} \mathrm{SOC}=-\frac{i_{b}}{Q_{\max }} .
$$

where $Q_{\max }$ is the battery capacity, and $i_{b}$ denotes the battery current, assumed positive during the discharge phase. The battery current can be solved with respect to $V_{o c}, R_{b}$, and the battery output power $P_{b}\left(=\left(V_{o c}-i_{b} R_{b}\right) i_{b}\right)$, by rearranging the $P_{b}$ expression, as follows:

$$
i_{b}=\frac{V_{o c}-\sqrt{V_{o c}^{2}-4 P_{b} R_{b}}}{2 R_{b}} .
$$

$V_{o c}$ is known in the literature to depend nonlinearly to the SOC, however here $V_{o c}$ is reasonably approximated by a constant voltage, which is compatible with the usual aim of a charge-sustaining (CS) battery management, by which the SOC is narrowly constrained; in the present work the battery $\mathrm{SOC}$ is limited within $\left[\mathrm{SOC}_{\min }, \mathrm{SOC}_{\max }\right]=[50 \%, 80 \%]$. Furthermore, by combining the battery with the bidirectional DC/DC converter, the SS output power is obtained by:

$$
P_{S S}=\eta_{d c}^{\operatorname{sign}\left(P_{S S}\right)} P_{b},
$$

in which $\eta_{d c}$ is the efficiency of the DC/DC converter. By applying the algebraic solution of $i_{b}$ (17) and the power balance equation (18) in (16), the dynamic behavior of the SS can be described by the differential equation of SOC with respect to $P_{S S}$ only:

$$
\frac{d}{d t} \mathrm{SOC}=\frac{-V_{o c}+\sqrt{V_{o c}^{2}-4 P_{S S} R_{b} / \eta_{d c}^{\operatorname{sign}\left(P_{S S}\right)}}}{2 R_{b} Q_{\max }} .
$$

b) Battery Thermal Model: The present work utilizes a control oriented single cell thermal model based on an active air cooling system, which represents a common solution for practical HEV battery systems. The lumped-parameter thermal model presented in [16], which assumes that all the cells have the same temperature and therefore the thermal interaction between them is ignored, is adapted in the present work to capture the essential thermal behavior of the battery with acceptable model complexity, by making the following further assumptions: 1) the cooling air temperature is uniform across the battery bank, and 2) the temperature across the cell is 
uniform. The first assumption enables a single cell model, while the second assumption is usually applied to reduce the model complexity by one dynamic state [36]; battery core and surface temperatures are lumped into a single variable, namely the cell temperature.

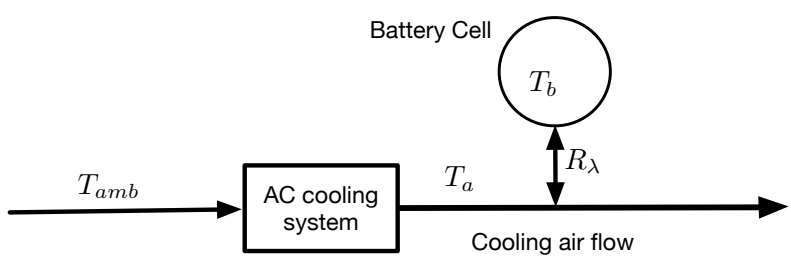

Fig. 6. The schematic diagram of the battery thermal management system.

The structure of the battery cooling system, described by the employed model, is illustrated in Fig 6. The battery cell thermal status in this case is described by the cell temperature $T_{b}$ and the cooling air temperature $T_{a}$. The dynamic of the cell temperature is governed by the following differential equation based on the heat convection:

$$
C_{b} \frac{d}{d t} T_{b}=\dot{Q}_{r}-\frac{T_{b}-T_{a}}{R_{\lambda}}
$$

where $\dot{Q}_{r}$ is the heat generation rate of a single battery cell induced by the cell resistance, $C_{b}$ is the heat capacity of the cell, and $R_{\lambda}$ is the thermal resistance between the cell and the cooling air. According to the layout of the cells, it is immediate to calculate: $\dot{Q}_{r}=R_{b} i_{b}^{2} / n$. The dynamic of the cooling air temperature is modeled as:

$$
C_{a} \frac{d}{d t} T_{a}=\frac{T_{b}-T_{a}}{R_{\lambda}}-\dot{Q}_{b, \text { cool }}
$$

where $\dot{Q}_{b, \text { cool }}$ is the cooling load for cooling one battery cell, and $C_{a}$ is the heat capacity of the air surrounding the single cell. Finally, $P_{C L B}$ is calculated by

$$
P_{C L B}=\frac{n \dot{Q}_{b, c o o l}}{\operatorname{COP} \eta_{i}}
$$

where COP is the coefficient of performance of the air conditioning system. In practice, the COP depends on several factors, including the refrigerant and ambient temperature. Inspired by test results of the R134a refrigerant published in [37], the COP is fixed at 2.25 for $T_{a m b}=30^{\circ} \mathrm{C}$.

\section{Optimal Control Problem Formulation}

The control objective in the present work is to minimize the overall fuel consumption for a driving mission by EM with consideration of propulsion and cooling requirements. Such requirements involve sustaining the battery SOC and maintaining the battery temperature within the optimum operating range:

$$
T_{b, \min } \leq T_{b} \leq T_{b, \max },
$$

with $T_{b, \text { min }}=15^{\circ} \mathrm{C}$ and $T_{b, \text { max }}=35^{\circ} \mathrm{C}$, while the coolant temperature at the engine's outlet and the radiator are requested to track reference temperatures for safe engine operation: $T_{e, t a r}=90^{\circ} \mathrm{C}, T_{r, t a r}=70^{\circ} \mathrm{C}$, respectively [16]. To implement the proposed joint propulsion and cooling EM strategy, the overall control problem is formulated in this section as an OCP. For benchmarking purposes, a conventional control strategy with separately controlled EM and TM systems is also introduced. The detailed formulation of both scenarios involves various OCPs, which will be specified in Sections III-A and III-B.

The OCPs formulated in the present work are solved by GPOPS-II [38], which is an optimal control software embedded in Matlab. GPOPS-II implements the Gaussian quadrature methods to convert an OCP into a sparse nonlinear programming problem (NLP), which is then tackled by the NLP solver IPOPT.

\section{A. Individual EM and TM control strategy (OCP-Split)}

The benchmarking strategy is a composite control scheme that combines conventional schemes for the various individual control tasks: a) conventional EM optimization for propulsion power only without considering the cooling systems, b) PI-control-based engine cooling, and c) TCS-based battery cooling. Although TCS represents the most commonly used technique for commercial vehicles, PI is selected here for engine temperature control as it offers more accurate tracking performance than the TCS.

1) Conventional EM optimization for propulsion power only $(O C P-E M)$ : The objective of this optimization problem is to find the primary and secondary sources power quantities, $P_{P S}$ and $P_{S S}$ respectively, that minimize the fuel consumption $m_{f}\left(t_{f}\right)$, subject to the powertrain system dynamics (fuel mass (7) and the SOC (19) only) and the algebraic constraint of power balance at the DC link as given by (2) (in this case $\left.P_{V L}=P_{P L}\right)$. The problem can be further simplified by utilizing: a) the constraint (2), b) the linear dependence of the fuel consumption rate on the PS power (see (7)), which enables the alternative objective of $\min \int_{t_{0}}^{t_{f}}\left(P_{P L}-P_{S S}\right) d t$ to be used instead of $\min m_{f}\left(t_{f}\right)$, and c) $\int_{t_{0}}^{t_{f}} P_{P L} d t$ being completely determined by the followed speed profile according to (1) and (6). OCP-EM can thus be simplified to an optimization problem with a single input $\mathbf{u}=P_{S S}$ and a single state $\mathbf{x}=\mathrm{SOC}$, as follows:

$$
\min _{P_{S S}}-\int_{t_{0}}^{t_{f}} P_{S S} d t
$$

subject to the dynamic system (19) and the following inequality constraints:

$\max \left(P_{P L}-P_{P S, \max }, P_{S S, \min }\right) \leq P_{S S} \leq \min \left(P_{S S, \max }, P_{P L}\right)$,

$\mathrm{SOC}_{\min } \leq \mathrm{SOC} \leq \mathrm{SOC}_{\max }$

where (24a) is inferred from (2) and the individual energy source power limits:

$$
0 \leq P_{P S} \leq P_{P S, \text { max }}, \quad P_{S S, \text { min }}<P_{S S}<P_{S S, \text { max }}
$$

The power limits are set to: $P_{P S, \max }=85 \mathrm{~kW}, P_{S S, \min }=$ $-16.5 \mathrm{~kW}$ and $P_{S S, \max }=24.6 \mathrm{~kW}$ (consequences of the C-rate limits specified in Table III). Finally, the following boundary conditions complete the problem:

$$
m_{f}\left(t_{0}\right)=0, \quad \operatorname{SOC}\left(t_{0}\right)=\operatorname{SOC}\left(t_{f}\right)=65 \% .
$$


in which the SOC boundary conditions ensure the battery to be CS at the end of the mission.

Given a driving cycle, the OCP-EM solves the $P_{S S}$ and consequently the power share profile: $\mu=P_{P S} / P_{P L}, \forall P_{P L}>0$ (when $P_{P L} \leq 0$, power split is fixed at $P_{P S}=0$ and $\left.P_{S S}=P_{P L}\right)$ that minimizes the overall fuel consumption for the hybrid powertrain without cooling systems.

2) OCP-Split: The first stage in OCP-Split is to solve OCPEM as described in Section III-A1 and obtain the power share trajectory $\mu$ for the given driving cycle. Subsequently, two PI controllers are deployed respectively for manipulating fan and pump speeds $\left(N_{f}\right.$ and $\left.N_{p}\right)$ to enforce the engine coolant temperatures $T_{e}$ and $T_{r}$ to track the predefined targets $T_{e, t a r}$ and $T_{r, \text { tar }}$, respectively, by using feedback based on the tracking-error. On the other hand, the battery cooling system controlled by the TCS is activated when $T_{b}$ hits the upper limit $T_{b, \max }$, and when $T_{b}$ goes back to $30^{\circ} \mathrm{C}$ the cooling system is switched off again. As soon as the battery cooling system is activated, the battery cooling power $P_{C L B}$ is operated at a constant load. The total power (propulsion, and cooling power determined by the PIs and TCS) requested to follow the driving cycle is met by the sum of $P_{P S}$ and $P_{S S}$ by following the power share trajectory $\mu$ found a priori by the OCP-EM for the same cycle. The fuel consumption and the SOC profile are finally evaluated by integrating (7) and (19).

\section{B. Joint propulsion and cooling EM (OCP-Joint)}

The OCP in this scenario is formulated for the full powertrain system to find its control inputs $\mathbf{u}$ that minimize the cost function $J(\mathbf{x}, \mathbf{u})$, taking multiple aspects into account, including fuel consumption, energy source thermal behavior and battery charge sustainability. The overall powertrain model that collects (19), (10)-(12) and (20)-(21) is represented in the following form:

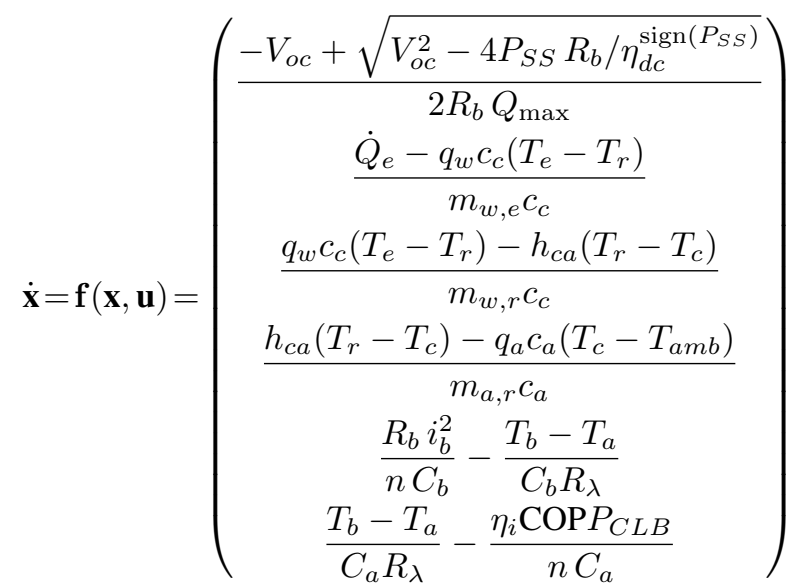

where $q_{w}, q_{a}, i_{b}, \dot{Q}_{e}$ can be explicitly expressed as functions of dynamic states $\mathbf{x}$ and control inputs $\mathbf{u}$, defined by: $\mathbf{x}=$ $\left[\mathrm{SOC}, T_{e}, T_{r}, T_{c}, T_{b}, T_{a}\right]^{\top}$ and $\mathbf{u}=\left[P_{S S}, N_{f}, N_{p}, P_{C L B}\right]^{\top}$. Similarly to the OCP-EM, $m_{f}$ and $P_{P S}$ are excluded from the state and control vectors to reduce the dimension of the dynamic model. When the optimal control inputs $\mathbf{u}$ are determined, $P_{P S}$ can be obtained immediately from (2)-(4) and (13)-(15). As such, the total fuel usage $m_{f}\left(t_{f}\right)$ is allowed to be evaluated a posteriori based on the optimum solution.
The multi-objective cost function proposed for this OCP, selected with further reduction in the complexity of the joint optimization problem in mind, is as follows:

$$
\begin{aligned}
& J(\mathbf{x}, \mathbf{u})=W_{1} \int_{t_{0}}^{t_{f}}\left(P_{C L E}+P_{C L B}-P_{S S}\right) d t \\
& +W_{2}\left(\operatorname{SOC}\left(t_{f}\right)-\operatorname{SOC}\left(t_{0}\right)\right)^{2}+W_{3} \int_{t_{0}}^{t_{f}}\left(T_{e}-T_{e, t a r}\right)^{2} d t \\
& +W_{4} \int_{t_{0}}^{t_{f}}\left(T_{r}-T_{r, \text { tar }}\right)^{2} d t+W_{5} \int_{t_{0}}^{t_{f}} \zeta\left(T_{b}\right) d t
\end{aligned}
$$

where the weighting factors $W_{k}, k=1,2, \cdots, 5$ are tuned to balance the control performance in the different aspects, with the first term of the cost function related to the fuel consumption, similarly to OCP-EM but now with the cooling power included. As it can be noticed, the second term represents a "soft constraint" for the CS operation over a full driving cycle. Such a formulation offers a suitable relaxation of the strictly $\mathrm{CS}$ condition $\mathrm{SOC}\left(t_{0}\right)=\mathrm{SOC}\left(t_{f}\right)$ (usually imposed as a boundary condition as shown in Section III-A for OCP-EM), with great benefit in terms of computation efficiency. The temperature tracking targets of the coolant at engine outlet and the radiator are addressed by quadratic cost functions of the tracking errors, formulated as the third and the fourth terms in (28). Moreover, it is worth noting that the last term in (28) formulates and includes in the objective function in a smooth manner the hard (path) constraint (23) on the battery temperature $T_{b}$, to facilitate the optimization algorithm. In particular, the following piecewise quadratic function is utilized in this framework to emulate the constraint (23):

$$
\zeta\left(T_{b}\right)=\left(\mu_{1} \tilde{T}_{b}^{2}+\mu_{2}\left\{\begin{array}{lr}
\left(\tilde{T}_{b}+H\right)^{2}, & \tilde{T}_{b} \leq-H \\
0, & -H<\tilde{T}_{b}<H \\
\left(\tilde{T}_{b}-H\right)^{2}, & \tilde{T}_{b} \geq H
\end{array}\right),\right.
$$

where $H=0.9, \mu_{1}=0.12, \mu_{2}=87.6$, and $\tilde{T}_{b}$ is the normalized temperature difference between $T_{b}$ and the mean of $T_{b, \max }$ and $T_{b, \min }$ :

$$
\tilde{T}_{b}=\frac{T_{b}-\frac{\left(T_{b, \max }+T_{b, \min }\right)}{2}}{\frac{\left(T_{b, \max }-T_{b, \min }\right)}{2}}=\frac{T_{b}-25}{10} .
$$

The behavior of (29) is illustrated in Fig. 7. It can be observed that the smoothed penalty function (29) highly resembles the ideal operational limits, being almost flat with 0 value within the acceptable working temperature $\left[15^{\circ} \mathrm{C}, 35^{\circ} \mathrm{C}\right]$.

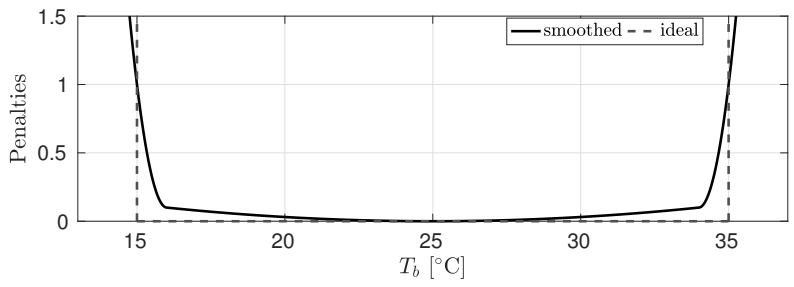

Fig. 7. Comparison of ideal and smoothed penalty function for $T_{b}$.

Similarly to the OCP-Split, the inequality constraints (24a) and (24b) are also enforced to keep the battery SOC and the power inputs from both energy sources inside their admissible 
range. In addition, the control inputs of the cooling systems are constrained by

$$
\begin{aligned}
& 0 \leq N_{f} \leq N_{f, \max }, \quad 0 \leq N_{p} \leq N_{p, \max }, \\
& 0 \leq P_{C L B} \leq P_{C L B, \max },
\end{aligned}
$$

with $N_{f, \max }=N_{p, \max }=6000 \mathrm{rpm}$ and $P_{C L B, \max }=6 \mathrm{~kW}$. Finally, dynamic states are initialized by the following conditions:

$$
\begin{array}{lc}
m_{f}\left(t_{0}\right)=0, & \operatorname{SOC}\left(t_{0}\right)=65 \%, \\
T_{e}\left(t_{0}\right)=T_{e, t a r}, & T_{r}\left(t_{0}\right)=T_{r, t a r}, \\
T_{b}\left(t_{0}\right)=35^{\circ} \mathrm{C}, & T_{c}\left(t_{0}\right)=T_{a}\left(t_{0}\right)=30^{\circ} \mathrm{C} .
\end{array}
$$

\section{Simulation Results}

\section{A. Driving Cycles}

The driving cycle defines the speed profile that needs to be followed by the vehicle. Thus, it heavily influences the operation of an EM strategy and the overall fuel economy. The present work utilizes a set of experimental driving speed profiles recorded respectively for urban, rural and motorway driving by a recently developed data acquisition device [39]. In contrast with artificial test cycles, these experimental profiles reflect realistic driving behavior where human drivers' inclination (e.g., usually to apply higher acceleration and deceleration than test cycles) and the effects of legal speed limits, road grades and traffic lights are naturally involved. The employed speed profiles are shown in Fig. 8, with some of their particular features specified in Table IV. The associated road elevation
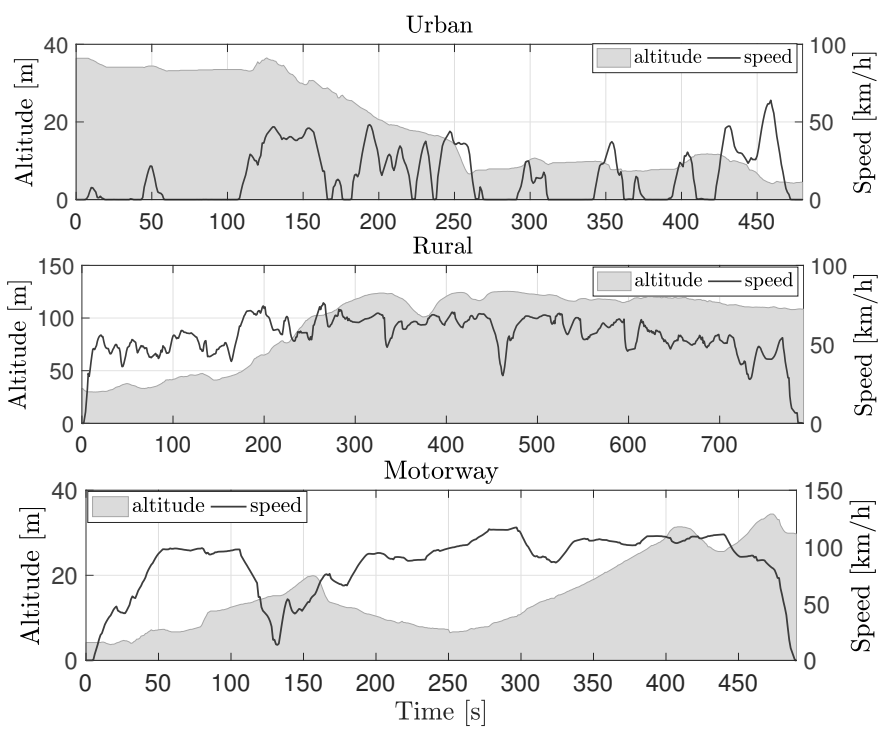

Fig. 8. Experimental speed profiles and the associated road elevation profiles for urban (top), rural (middle) and motorway (bottom) driving.

data of the experimental driving missions is also measured and converted to the slope $\theta$, which contributes to the total driving force via $m_{v} g \sin \theta$ and $f_{T} m_{v} g \cos \theta$ (see (1)).

\section{B. Numerical Results}

Simulation examples are carried out in this Section based on the experimental driving cycles shown in Fig. 8 with the aim to: 1) evaluate the cooling losses in different and practical
TABLE IV

CHARACTERISTICS OF EXPERIMENTAL SPEED PROFILES

\begin{tabular}{lccc}
\hline \hline & Urban & Rural & Motorway \\
\hline Duration [s] & 480 & 792 & 490 \\
Distance [km] & 1.99 & 12.17 & 11.66 \\
Average Speed [km/h] & 14.9 & 55.2 & 85.5 \\
Max speed [km/h] & 63.9 & 76.3 & 117.2 \\
Max acceleration [m/s $\left.{ }^{2}\right]$ & 3.87 & 2.77 & 2.33 \\
Max deceleration $\left[\mathrm{m} / \mathrm{s}^{2}\right]$ & -3.42 & -3.64 & -3.44 \\
\hline \hline
\end{tabular}

driving scenarios, 2) analyze how the cooling load changes the optimal EM solutions, and 3) show the benefits of the proposed OCP-Joint over the conventional composite control strategy, OCP-Split, in terms of fuel savings and other aspects. To this end, the optimal solutions solved by two realizations of OCP-Joint (OCP-Joint A and OCP-Joint B), with a different tuning parameter set for each realization, are benchmarked against the results of OCP-Split, at $30^{\circ} \mathrm{C}$ ambient temperature. The choice of realization A aims to assess the capability of OCP-Joint to track engine and radiator coolant temperatures more accurately than with OCP-Split, while maintaining the same fuel consumption as OCP-Split. The introduction of realization $\mathrm{B}$ aims to assess the improvement of OCP-Joint in fuel economy as compared to OCP-Split, while OCP-Joint maintains a similar engine and radiator coolant temperature tracking precision as with OCP-Split. Note that the CS condition $\operatorname{SOC}\left(t_{0}\right)=\operatorname{SOC}\left(t_{f}\right)$ is not guaranteed when the OCPSplit is employed, since it is imposed only in the stage of OCP-EM. However, in addition to the fuel consumption and temperature tracking, as mentioned above, the terminal SOC of the two OCP-Joint approaches can be freely assigned by tuning the weights in (28). To ensure a fair comparison, the terminal SOC conditions, $\mathrm{SOC}\left(t_{f}\right)$, of the two OCP-Joint schemes in each driving cycle case are enforced to be identical to the terminal SOC reached in the results obtained by OCP-Split.

a) OCP-Split: The PI controllers and TCS of the OCPSplit are tuned, as shown in Table V, such that the tracking error satisfies:

$$
\begin{array}{rlrl} 
& \left|T_{e}-T_{e, t a r}\right| \leq 1.5^{\circ} \mathrm{C}, & & \left|T_{r}-T_{r, t a r}\right| \leq 3^{\circ} \mathrm{C}, \\
\left|T_{e}-T_{e, t a r}\right| \leq 2^{\circ} \mathrm{C}, & & \left|T_{r}-T_{r, t a r}\right| \leq 4^{\circ} \mathrm{C}, \\
\left|T_{e}-T_{e, t a r}\right| \leq 2^{\circ} \mathrm{C}, & & \left|T_{r}-T_{r, t a r}\right| \leq 8^{\circ} \mathrm{C},
\end{array}
$$

respectively for urban, rural and motorway driving situations (it is expected that the OCP-Split tracking accuracy can be improved at the price of more fuel usage as the PI tuning is subject to a trade-off between tracking accuracy and control power). The approach followed is to tune by trial and error the PI control and TCS gains and parameters, such that for a target fuel consumption the best tracking performance is achieved.

b) OCP-Joint A: In this case the OCP-Joint weights in (28) are selected as shown in Table V, such that the resulting fuel consumption and the terminal SOC are the same as in the solutions of OCP-Split in each driving cycle case respectively, to enable a first fair comparison between OCP-Joint and OCPSplit in terms of temperature tracking accuracy.

c) OCP-Joint B: In this case the OCP-Joint coolant temperature tracking precision is relaxed as compared to OCP-Joint A by adjusting the weights in (28), as shown in 
Table V, such that OCP-Joint exhibits a similar engine and radiator coolant temperature behavior (and peak variation), while maintaining the same $\operatorname{SOC}\left(t_{f}\right)$, as OCP-Split. Thus, a further fair comparison between OCP-Joint and OCP-Split is enabled, in this case in terms of fuel economy.

TABLE V

WEIGHTING FACTORS OF THE OCP-JOINT OBJECTIVE FUNCTION (28) AS WELL AS PI AND TCS PARAMETERS OF THE OCP-SPLIT

\begin{tabular}{lccccc}
\hline \hline & $W_{1}$ & $W_{2}$ & $W_{3}$ & $W_{4}$ & $W_{5}$ \\
\hline OCP-Joint A (Urban) & 5.5 & $5 \mathrm{e} 3$ & $2 \mathrm{e}-2$ & $1 \mathrm{e}-3$ & $2.5 \mathrm{e}-3$ \\
OCP-Joint A (Rural) & 12 & $5 \mathrm{e} 3$ & $2 \mathrm{e}-2$ & $1 \mathrm{e}-3$ & $2.5 \mathrm{e}-3$ \\
OCP-Joint A (Motorway) & 4 & $5 \mathrm{e} 3$ & $2 \mathrm{e}-2$ & $1 \mathrm{e}-3$ & $2.5 \mathrm{e}-3$ \\
OCP-Joint B (Urban) & 10 & $5 \mathrm{e} 3$ & $2 \mathrm{e}-5$ & $1.5 \mathrm{e}-6$ & $2.5 \mathrm{e}-3$ \\
OCP-Joint B (Rural) & 10 & $5 \mathrm{e} 3$ & $1.6 \mathrm{e}-6$ & $6 \mathrm{e}-7$ & $1.65 \mathrm{e}-3$ \\
OCP-Joint B (Motorway) & 10 & $5 \mathrm{e} 3$ & $2 \mathrm{e}-5$ & $1.5 \mathrm{e}-6$ & $2.5 \mathrm{e}-3$ \\
\hline \multicolumn{2}{c}{$\mathrm{PI}$ for the pump } & $\mathrm{PI}$ for the fan & TCS operating power \\
\hline Urban & $\mathrm{P}=800, \mathrm{I}=2$ & $\mathrm{P}=750, \mathrm{I}=1$ & \multicolumn{2}{c}{$1 \mathrm{~kW}$} \\
Rural & $\mathrm{P}=750, \mathrm{I}=5$ & $\mathrm{P}=700, \mathrm{I}=2$ & \multicolumn{2}{c}{$1 \mathrm{~kW}$} \\
Motorway & $\mathrm{P}=800, \mathrm{I}=8$ & $\mathrm{P}=700, \mathrm{I}=0.5$ & \multicolumn{3}{c}{} \\
\hline \hline
\end{tabular}

1) Comparison between OCP-Joint A and OCP-Split: In this subsection the behavior and performance of OCP-Joint A are compared and benchmarked against those of OCP-Split, firstly in terms of powertrain and cooling power flows, subsequently in terms of engine coolant and battery temperature response as well as battery SOC variation, and finally in terms of average energy source and cooling power losses including power balance validation.

Figures 9-11 compare the optimal power profiles in different driving situations, where it is shown that the inclusion of the cooling systems in the EM optimization (that places emphasis on coolant temperature tracking) changes the optimal power split pattern between the two energy sources. As it can be

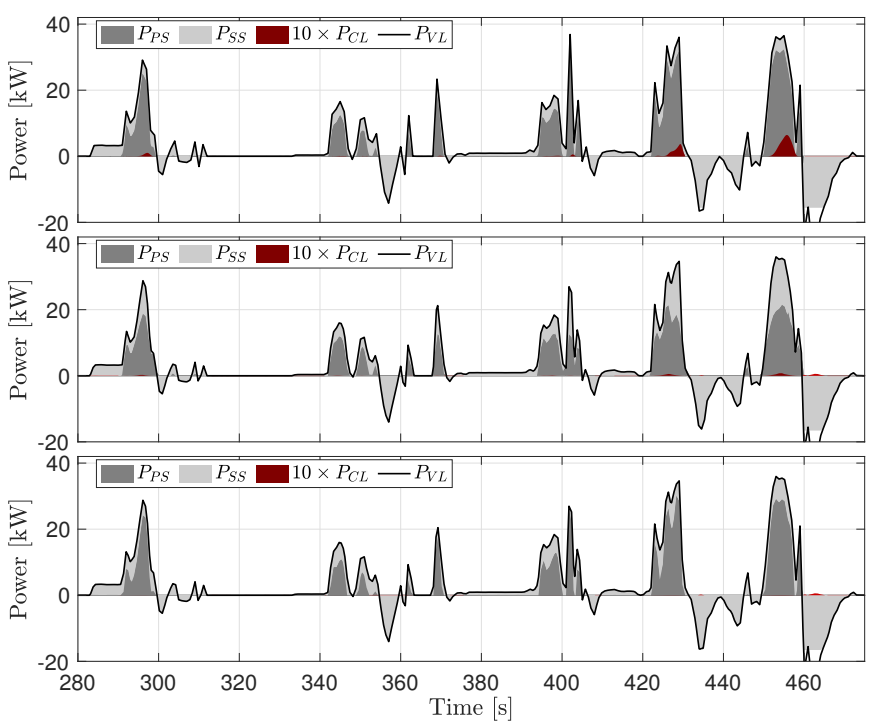

Fig. 9. Power flows $P_{V L}, P_{P S}$ and $P_{S S}$ solved by OCP-Split (top), OCPJoint A (middle) and OCP-Joint B (bottom) for urban driving (a part of the driving cycle is shown). The amount of total cooling power $P_{C L}$ is multiplied by 10 and also overlaid.

seen, the optimal solution of the OCP-Split in each driving cycle case operates the SS as an energy buffer that provides only a small amount of power for propulsion (and proportionally for cooling according to power share $\mu$ ), while the PS provides

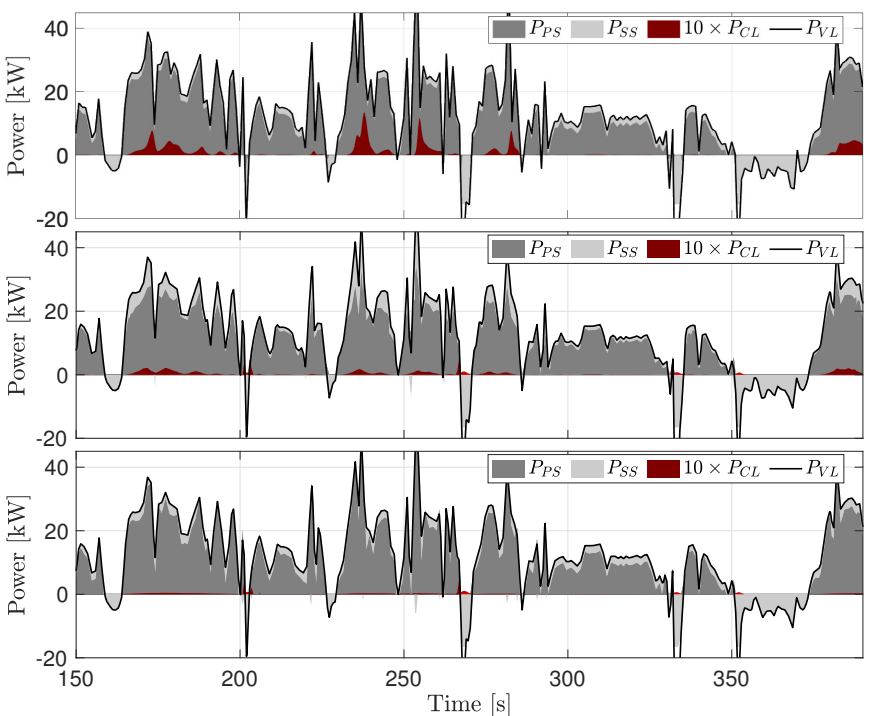

Fig. 10. Power flows $P_{V L}, P_{P S}$ and $P_{S S}$ solved by OCP-Split (top), OCPJoint A (middle) and OCP-Joint B (bottom) for rural driving (a part of the driving cycle is shown). The amount of total cooling power $P_{C L}$ is multiplied by 10 and also overlaid.
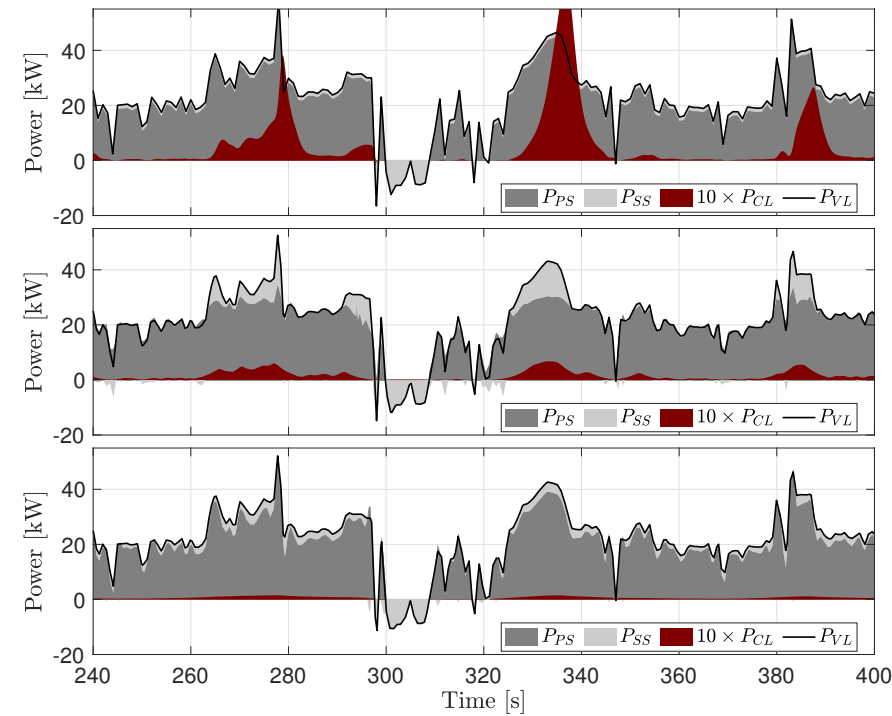

Fig. 11. Power flows $P_{V L}, P_{P S}$ and $P_{S S}$ solved by OCP-Split (top), OCP-Joint A (middle) and OCP-Joint B (bottom) for motorway driving (a part of the driving cycle is shown). The amount of total cooling power $P_{C L}$ is multiplied by 10 and also overlaid.

the rest of the power. Conversely, since the OCP-Joint A solution is strongly influenced by the combined emphasis on reducing both cooling power (related to the fuel consumption objective) and variations of ICE coolant temperatures from given setpoints, the PS power is suppressed to avoid high PS power output, and much more SS power is applied to meet high power demands. At the same time, in order to compensate the increased SS usage, the OCP-Joint A often increases the PS usage when the power request is small (for example, approximately at $308-324 \mathrm{~s}$ in motorway driving), so that the battery can be charged through the engine to maintain charge sustaining operation, resulting in a somewhat "flat" PS power profile for the whole mission; this is more visible in the case of motorway driving. This behavior can be attributed to: a) 
the fact that it is easier to enforce steady coolant temperatures when the ICE power is steady, and b) the cubic law governing the engine cooling power (see (14)) as opposed to the linear law for the battery cooling power (see (22)), such that engine cooling is much more costly than battery cooling at high cooling loads. It is clear from Figs. 9-11, therefore, that the total cooling power is significantly reduced by OCP-Joint A, with further insight on the mechanism by which this happens given in Fig. 12 which provides the breakdown of cooling power components in an exemplary case. In this figure the
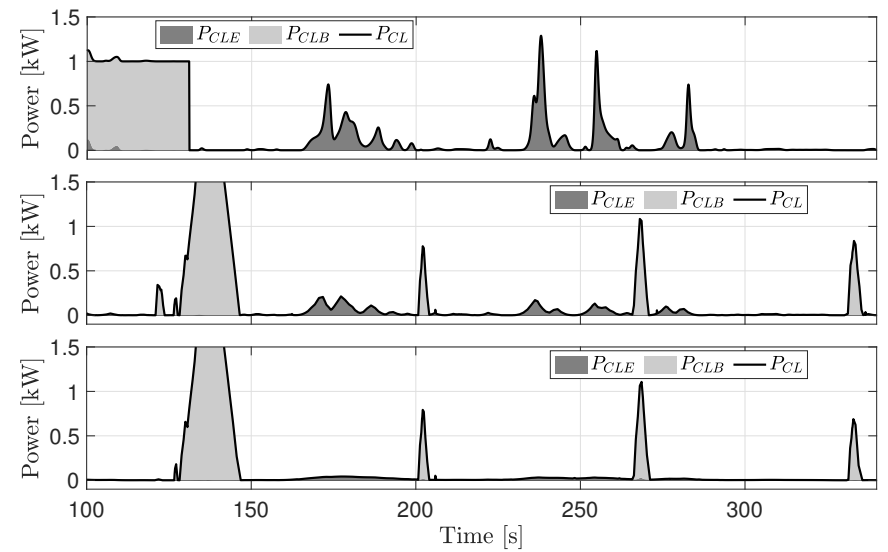

Fig. 12. Cooling power flows $P_{C L}, P_{C L E}$ and $P_{C L B}$ solved by OCP-Split (top), OCP-Joint A (middle) and OCP-Joint B (bottom) for rural driving (a part of the driving cycle is shown).

engine and battery cooling power profiles for the rural driving OCP-Split and OCP-Joint A cases shown for in Fig. 10 are illustrated. It can be seen that the large peaks of engine cooling power and low levels of battery cooling power in OCP-Split are replaced with a combination of significantly lower levels of engine cooling power and more spread battery cooling power in OCP-Joint A.

The thermal and SOC performance of OCP-Joint A and OCP-Split is compared in Figs. 13-14. As it can be seen, when OCP-Joint $\mathrm{A}$ is employed, the target temperatures of the engine coolant are accurately followed, whereas the PI control in the engine cooling system (OCP-Split) is unable to provide comparable temperature tracking accuracy as the joint optimization solution OCP-Joint A, as also shown by (32). Moreover, the battery temperature, $T_{b}$, of both OCP schemes is kept within the desired range throughout the mission in all driving cycles. The CS condition $\operatorname{SOC}\left(t_{0}\right)=\operatorname{SOC}\left(t_{f}\right)$ of OCP-Split is imposed only in the first stage OCP-EM whose optimal solutions solely charge the battery from regenerative braking and not by any direct charging from the PS [40]. Therefore, when the power split is applied according to the $\mu$ trajectory, the additional cooling power drives the battery to be less charged: $\operatorname{SOC}\left(t_{f}\right)<\operatorname{SOC}\left(t_{0}\right)$. The resulting SOC terminal condition of OCP-Split is targeted by OCP-Joint A, as illustrated in Fig. 14. Hence, it is guaranteed that both OCP schemes consume the same amount of energy, with consideration of fuel and battery charge.

The average energy source and cooling power losses for the whole mission in each driving cycle case are also evaluated for each OCP, which reinforce some of the earlier observations
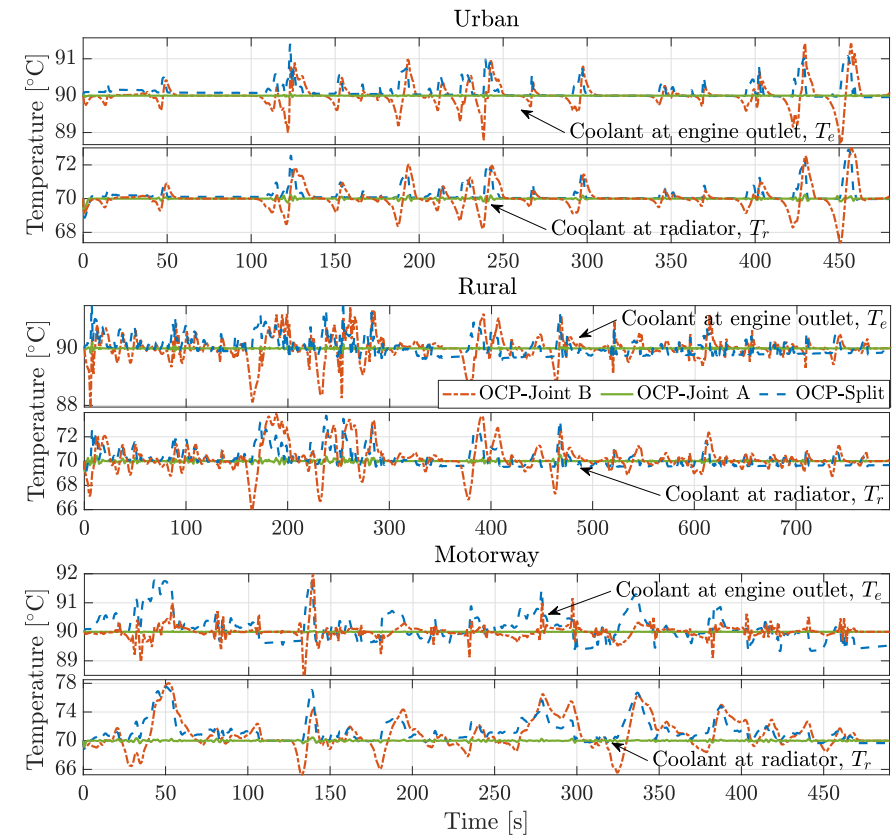

Fig. 13. Temperature of coolant at engine outlet, $T_{e}$, and radiator, $T_{r}$, solved by OCP-Split (dashed), OCP-Joint A (solid) and OCP-Joint B (dash dot) for urban (top), rural (middle), and motorway (bottom) driving.
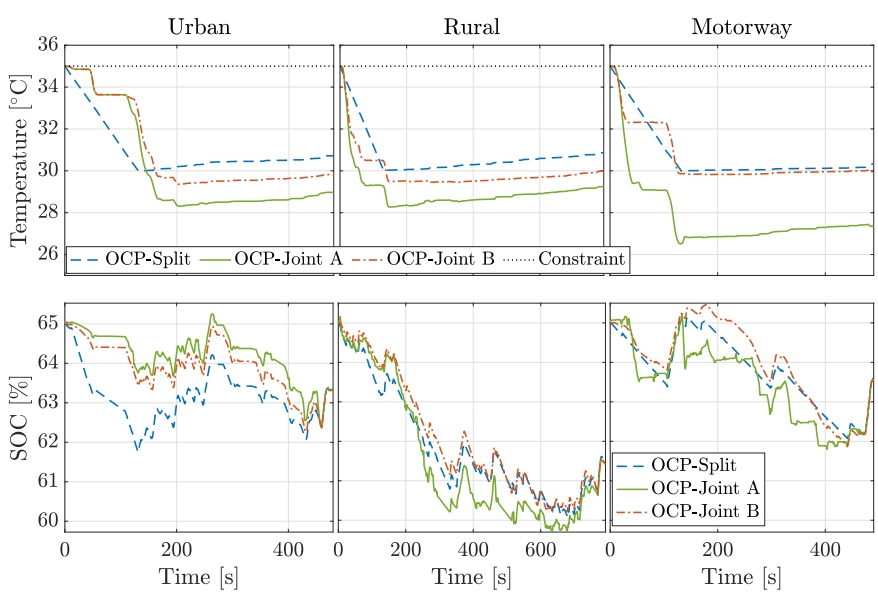

Fig. 14. Battery temperature, $T_{b}$, and SOC profiles solved by OCP-Split (dashed), OCP-Joint A (solid) and OCP-Joint B (dash dot) for urban (left), rural (middle), and motorway (right) driving.

and conclusions, and also provide further and more complete insight about the nature of the various OCP solutions. In particular, the average PS branch power loss is computed by $\frac{1}{t_{f}-t_{0}} \int_{t_{0}}^{t_{f}}\left(P_{f}-P_{P S}\right) d t$, while the loss of the SS branch is determined by $\frac{1}{t_{f}-t_{0}} \int_{t_{0}}^{t_{f}}\left|V_{o c} i_{b}-P_{S S}\right| d t$, which takes both charging and discharging losses into account. Similarly, the average cooling power for the ICE and the battery is obtained by $\frac{1}{t_{f}-t_{0}} \int_{t_{0}}^{t_{f}} P_{C L E} d t$ and $\frac{1}{t_{f}-t_{0}} \int_{t_{0}}^{t_{f}} P_{C L B} d t$ respectively. The histogram in Fig. 15 shows the aforementioned power losses for each OCP in different driving scenarios. It is clear that the vast majority of losses in all simulated cases corresponds to PS branch power losses, which is expected since the most inefficient component of the powertrain is the ICE with peak efficiencies of 0.36, as shown in Fig. 3. The first important observation, which also serves as an overall powertrain power balance validation, is that the OCP-Split and OCP-Joint A 


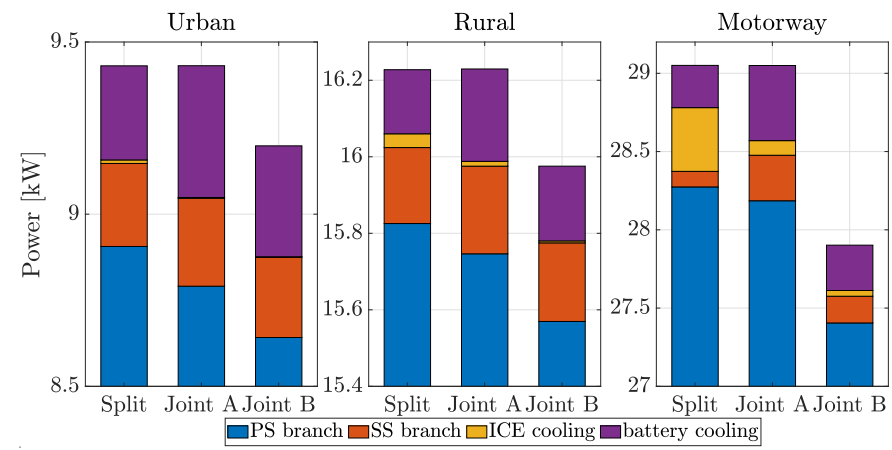

Fig. 15. Average energy source and cooling power losses solved by OCPSplit, OCP-Joint A and OCP-Joint B in each driving cycle case. The numerical values are obtained by dividing the overall energy values for the whole mission with the duration of the drive cycle in each case. For clarity, only the top part of each bar is shown, in which the blue PS branch average power loss starts from $0 \mathrm{~kW}$ in all cases.

bars have the same total height in all driving cycle cases, as would be expected from the design choice of identical fuel consumption and SOC variation throughout the mission in both OCPs. The proportion of each type of loss, however, is different between the two OCP cases. As more SS power is used in OCP-Joint A to eliminate peaks of PS power (see Figs. 9-11), the SS and PS branch power losses are respectively increased and decreased. There is a similar change in the associated cooling power losses. These observations are true for all driving cycle cases.

2) Comparison between $O C P$-Joint $B$, and OCP-Joint $A$ and OCP-Split: OCP-Joint B is shown in Fig. 13 to track the engine and radiator coolant temperature set points in a similar manner to OCP-Split, according to the choice of the weights of (28) shown in Table V. In terms of battery temperature profiles shown in Fig. 14, OCP-Joint B yields more similar solutions to OCP-Split as compared to the solutions of OCP-Joint A. Figure 14 also verifies that the terminal SOC conditions obtained by OCP-Split are reached by OCP-Joint B in all examples.

Since OCP-Joint B puts the emphasis on fuel consumption reduction, as with OCP-EM, the optimal power sharing of OCP-Joint B has a similar pattern to the OCP-Split solutions, but with moderately higher and lower usage of SS and PS power respectively, as shown by the power profiles in Figs. 911. As such, the total energy source power losses (sum of SS and PS branch power losses) of OCP-Joint B are reduced as compared to both OCP-Split and OCP-Joint A, as shown in Fig. 15, since PS branch power comes at a much lower efficiency than SS branch power. Furthermore, this moderate change in the power sharing balance serves to reduce considerably the PS cooling power losses with only marginal increases in SS cooling power losses of the OCP-Joint B as compared to OCP-Split, while both types of cooling losses are reduced by OCP-Joint B as compared to OCP-Joint A, as also shown in Fig. 15 and Fig. 12. Hence, the total cooling power losses of OCP-Joint B become significantly less compared to OCPSplit, as it can be seen in Figs. 9-11. Thus, the overall losses of OCP-Joint B are the lowest as compared to the other two OCPs, which is also reflected in the fuel economy. By making use of the density of petroleum diesel, $0.832 \mathrm{~kg} / \mathrm{L}$, and the length of each driving cycle (see Table IV), the fuel economy of OCP-Joint B and OCP-Split is calculated and compared in Table VI. As shown, the simultaneous optimization mechanism

TABLE VI

EVALUATION OF FUEL ECONOMY [L/100KM] AND PERCENTAGE OF FUEL SAVING OF OCP-JOINT B AS COMPARED TO OCP-SPLIT

\begin{tabular}{lccc}
\hline \hline & OCP-Joint B & OCP-Split & Fuel saving \\
\hline Urban & 7.83 & 8.00 & $2.12 \%$ \\
Rural & 4.24 & 4.27 & $0.7 \%$ \\
Motorway & 5.09 & 5.22 & $2.49 \%$ \\
\hline \hline
\end{tabular}

OCP-Joint B is able to save $2.12 \%, 0.7 \%$ and $2.49 \%$ fuel usage respectively for the urban, rural and motorway cycles as compared to the conventional composite strategy OCP-Split.

Finally, the differences in cooling power losses among the three driving scenarios are examined for OCP-Joint $\mathrm{A}$ and OCP-Joint B in Fig. 15, with similar observations in both OCPs. Battery cooling is dominant when following the urban speed profile. As the speed increases, more ICE cooling is required. Typically, ICE cooling dominates for OCP-Split solution when the speed increases further to motorway driving. The overall cooling power requirements increase for all OCPs as the driving speed increases from low speed (urban) to high speed (motorway) driving. Nevertheless, rural driving is amenable to be optimized to the lowest total cooling power consumption, as compared to the urban and motorway speed profiles. This can be associated with the almost constant speed cruising about the average speed in the rural driving style employed in this work, which is a more efficient driving pattern in terms of cooling.

\section{CONCLUDING REMARKS}

The joint energy and thermal management control of a series hybrid electric vehicle is addressed in this paper by an optimal control methodology. The optimal control problem is formulated by suitably integrating the battery and the internal combustion engine thermal models to a conventional powertrain model without significantly increasing the overall model complexity. A weighted multi-objective cost function of the various control performance aspects is formulated with a reduction in optimization complexity in mind. As such, the proposed joint energy management (EM) control strategy is able to find the appropriate power split between the engine and battery, which minimizes the overall fuel consumption while fulfilling the cooling requirements of both energy sources. In this paper, the numerical case studies are carried out in the context of three real-world speed profiles obtained experimentally in urban, rural and motorway driving scenarios. The importance of including the thermal dynamics in the EM problem is illustrated by numerical comparisons with the existing EM optimization strategy that neglects the thermal dynamics and optimizes on the basis of propulsion power only, while employing traditional PI and thermostat controlbased cooling systems. It is found that the battery plays a more active role when the cooling losses are considered in the EM optimization since the engine cooling system is less efficient than the battery cooling system at high cooling loads. By employing two tuning sets of the optimization objective 
function, the benefit of the proposed strategy is shown both in terms of enhanced cooling systems temperature control tracking as well as fuel economy. With the cooling system temperature tracking performance tuned to be equivalent to that of the conventional method, the joint propulsion and cooling energy management optimization method is found to outperform the conventional approach for all studied driving cycles with up to a $2.49 \%$ improvement in terms of fuel consumption.

\section{REFERENCES}

[1] L. Serrao, S. Onori, and G. Rizzoni, "A comparative analysis of energy management strategies for hybrid electric vehicles," Journal of Dynamic Systems, Measurement, and Control, vol. 133, no. 3, 2011.

[2] C. Hou, M. Ouyang, L. Xu, and H. Wang, "Approximate pontryagin's minimum principle applied to the energy management of plug-in hybrid electric vehicles," Applied Energy, vol. 115, pp. 174-189, 2014.

[3] S. Uebel, N. Murgovski, C. Tempelhahn, and B. Baker, "Optimal energy management and velocity control of hybrid electric vehicles," IEEE Transactions on Vehicular Technology, vol. 67, no. 1, pp. 327-337, 2018.

[4] A. A. Malikopoulos, "Supervisory power management control algorithms for hybrid electric vehicles: A survey," IEEE Transactions on Intelligent Transportation Systems, vol. 15, no. 5, pp. 1869-1885, 2014

[5] S. G. Wirasingha and A. Emadi, "Classification and review of control strategies for plug-in hybrid electric vehicles," IEEE Transactions on Vehicular Technology, vol. 60, no. 1, pp. 111-122, 2011.

[6] C. M. Martinez, X. Hu, D. Cao, E. Velenis, B. Gao, and M. Wellers, "Energy management in plug-in hybrid electric vehicles: Recent progress and a connected vehicles perspective," IEEE Transactions on Vehicular Technology, vol. 66, no. 6, pp. 4534-4549, 2017.

[7] J. Zhang and T. Shen, "Real-time fuel economy optimization with nonlinear mpc for phevs," IEEE Transactions on Control Systems Technology, vol. 24, no. 6, pp. 2167-2175, 2016.

[8] Y. Zhang, H. Liu, and Q. Guo, "Varying-domain optimal management strategy for parallel hybrid electric vehicles," IEEE Transactions on Vehicular Technology, vol. 63, no. 2, pp. 603-616, 2014.

[9] W. Shabbir and S. A. Evangelou, "Threshold-changing control strategy for series hybrid electric vehicles," Applied Energy, pp. 761-775, 2019.

[10] V. H. Johnson, K. B. Wipke, and D. J. Rausen, "Hev control strategy for real-time optimization of fuel economy and emissions," SAE Technical Paper 2000-01-1543, 2000.

[11] S. Uebel, N. Murgovski, C. Tempelhahn, and B. Baker, "A two-level $\mathrm{mpc}$ for energy management including velocity control of hybrid electric vehicles," IEEE Transactions on Vehicular Technology, vol. 68, no. 6, pp. 5494-5505, 2019.

[12] J. Liu, Y. Chen, J. Zhan, and F. Shang, "Heuristic dynamic programming based online energy management strategy for plug-in hybrid electric vehicles," IEEE Transactions on Vehicular Technology, vol. 68, no. 5, pp. 4479-4493, 2019.

[13] G. Ma, M. Ghasemi, and X. Song, "Integrated powertrain energy management and vehicle coordination for multiple connected hybrid electric vehicles," IEEE Transactions on Vehicular Technology, vol. 67, no. 4, pp. 2893-2899, 2018.

[14] R. Schmid, J. Burger, and N. Bajcinca, "Efficient optimal control of plug-in-hybrid electric vehicles including explicit engine on/off decisions," in European Control Conference, 2018, pp. 596-601.

[15] M. H. Salah, T. H. Mitchell, J. R. Wagner, and D. M. Dawson, "Nonlinear-control strategy for advanced vehicle thermal-management systems," IEEE Transactions on Vehicular Technology, vol. 57, no. 1, pp. 127-137, 2008 .

[16] X. Tao, K. Zhou, A. Ivanco, J. R. Wagner, H. Hofmann, and Z. Filipi, “A hybrid electric vehicle thermal management system-nonlinear controller design," SAE Technical Paper 2015-01-1710, 2015.

[17] A. Y. Karnik, A. Fuxman, P. Bonkoski, M. Jankovic, and J. Pekar, "Vehicle powertrain thermal management system using model predictive control," SAE International Journal of Materials and Manufacturing, vol. 9, no. 3, pp. 525-533, 2016.

[18] S. Park and D. Jung, "Numerical modeling and simulation of the vehicle cooling system for a heavy duty series hybrid electric vehicle," $S A E$ Technical Paper 2008-01-2421, 2008.

[19] J. K. Carroll, M. Alzorgan, C. Page, and A. R. Mayyas, "Active battery thermal management within electric and plug-in hybrid electric vehicles," SAE Technical Paper 2016-01-2221, 2016.
[20] F. Caresana, M. Bilancia, and C. Bartolini, "Numerical method for assessing the potential of smart engine thermal management: Application to a medium-upper segment passenger car," Applied Thermal Engineering, vol. 31, no. 16, pp. 3559-3568, 2011.

[21] M. Shams-Zahraei, A. Z. Kouzani, S. Kutter, and B. Baker, "Integrated thermal and energy management of plug-in hybrid electric vehicles," Journal of Power Sources, vol. 216, pp. 237-248, 2012.

[22] H. Pham, P. Van Den Bosch, J. Kessels, and R. Huisman, "Integrated energy and thermal management for hybrid electric heavy duty trucks," in IEEE Vehicle Power and Propulsion Conference, 2012, pp. 932-937.

[23] J. Lescot, A. Sciarretta, Y. Chamaillard, and A. Charlet, "On the integration of optimal energy management and thermal management of hybrid electric vehicles," in IEEE Vehicle Power and Propulsion Conference, 2010, pp. 1-6.

[24] X. Zhang, A. Ivanco, X. Tao, J. Wagner, and Z. Filipi, "Optimization of the series-hev control with consideration of the impact of battery cooling auxiliary losses," SAE Int. J. Alt. Power, vol. 3, no. 2, pp. 234-243, 2014.

[25] V. van Reeven, R. Huisman, J. Kessels, H. Pham, and T. Hofman, "Integrating energy and thermal management of hybrid trucks," in Proceedings of the 12th International Conference Commercial Vehicles Trucks, Bus, Van, Trailer, 2013, pp. 5-6.

[26] F. J. Jimenez-Espadafor, D. P. Guerrero, E. C. Trujillo, M. T. Garcia, and J. Wideberg, "Fully optimized energy management for propulsion, thermal cooling and auxiliaries of a serial hybrid electric vehicle," Applied Thermal Engineering, vol. 91, pp. 694-705, 2015.

[27] X. Li, S. A. Evangelou, and R. Lot, "Integrated management of powertrain and engine cooling system for parallel hybrid electric vehicles," in IEEE Vehicle Power and Propulsion Conference, 2018.

[28] R. Lot and S. A. Evangelou, "Green driving optimization of a series hybrid electric vehicle," in 52nd IEEE Conference on Decision and Control, 2013, pp. 2200-2207.

[29] W. Zhou, C. Zhang, J. Li, and H. K. Fathy, "A pseudospectral strategy for optimal power management in series hybrid electric powertrains," IEEE Transactions on Vehicular Technology, vol. 65, no. 6, pp. 4813$4825,2016$.

[30] P. Setlur, J. R. Wagner, D. M. Dawson, and E. Marotta, "An advanced engine thermal management system: Nonlinear control and test," IEEE/ASME Transactions on Mechatronics, vol. 10, no. 2, pp. 210-220, 2005.

[31] T. Markel, A. Brooker, T. Hendricks, V. Johnson, K. Kelly, B. Kramer, M. O'Keefe, S. Sprik, and K. Wipke, "Advisor: a systems analysis tool for advanced vehicle modeling," Journal of Power Sources, vol. 110, no. 2, pp. 255-266, 2002

[32] S. Chu and A. Majumdar, "Opportunities and challenges for a sustainable energy future," Nature, vol. 488, pp. 294-303, 2012.

[33] T. T. Wang and J. Wagner, "Advanced automotive thermal managementnonlinear radiator fan matrix control," Control Engineering Practice, vol. 41, pp. 113-123, 2015 .

[34] M. H. Salah, T. H. Mitchell, J. R. Wagner, and D. M. Dawson, "A smart multiple-loop automotive cooling system - model, control, and experimental study," IEEE/ASME Transactions on Mechatronics, vol. 15, no. 1 , pp. 117-124, 2009.

[35] X. Tao, "Design, modeling and control of a thermal management system forhybrid electric vehicles," Ph.D. dissertation, Clemson University, May 2016.

[36] M. Salameh, S. Wilke, S. A.-H. B. Schweitzer, P. Sveum, and M. Krishnamurthy, "Thermal state of charge estimation in phase change composites for passively cooled lithium-ion battery packs," IEEE Transactions on Industry Applications, vol. 54, no. 1, pp. 426-436, 2018.

[37] J. Brown, S. F.Yana-Motta, and P. A.Domanski, "Comparitive analysis of an automotive air conditioning systems operating with $\mathrm{Co}_{2}$ and r134a," International Journal of Refrigeration, vol. 25, pp. 19-32, 2002.

[38] A. V. Rao, D. Benson, C. Darby, M. Patterson, C. Francolin, I. Sanders, and G. Huntington, "Gpops: A matlab software for solving multiplephase optimal control problems using the gauss pseudospectral method,' ACM Transactions on Mathematical Software, vol. 37, no. 2, 2010.

[39] X. Yan, J. Fleming, C. Allison, and R. Lot, "Portable automobile data acquisition module (adam) for naturalistic driving study," in 15th EAEC European Automotive Congress, 2017.

[40] B. Chen and S. A. Evangelou, "Truncated battery power following strategy for energy management control of series hybrid electric vehicles," in IEEE European Control Conference, 2019, pp. 738-743. 